\title{
Principais causas de morte neonatal em Criciúma e o papel do enfermeiro quanto a preveni-las
}

\author{
Aline Gabriel Lima*, Cecília Marly Spiazzi dos Santos, M.Sc.**, Gabriela Acordi da Silva Poluceno**, \\ Maria Tereza Soratto, M.Sc.**, Luciane Bisognin Ceretta***
}

Acadêmica do Curso de Enfermagem da Universidade do Extremo Sul Catarinense (UNESC), Criciúma/SC, **Enfermeira, Professora do Curso de Enfermagem da UNESC, Criciúma/SC, ***Enfermeira, Doutoranda em Ciências da Saúde, Professora do Curso de Enfermagem da UNESC, Criciuma/SC

\section{Resumo}

De acordo com o Ministério da Saúde a mortalidade neonatal passou a ser o principal componente de mortalidade infantil a partir do final da década de 80. Considerando tal fato, o objetivo do presente estudo foi identificar as principais causas de mortalidade neonatal no município de Criciúma/SC entre 2006 e 2010, e compreender o papel do enfermeiro frente à prevenção dessas mortes. Os dados foram coletados analisando declaraçóes de óbito; dados do Sistema de Informaçôes sobre Mortalidade (SIM); e dos prontuários das gestantes nas Unidades de Saúde onde realizaram o pré-natal. Verificou-se que ocorreram 119 óbitos neonatais com residentes de Criciúma, sendo 72,2\% ocorridos no período neonatal precoce e 27,7\% no período neonatal tardio. Foram identificadas 47 causas diferenciadas, sendo $64 \%$ delas consideradas evitáveis por intervençóes no âmbito do SUS. Analisando os prontuários das gestantes, 44,6\% não foram encontrados; 29,8\% só possuíam anotaçôes de outros profissionais; e 25,6\% não possuíam evolução. A realizaçáo do estudo foi de grande aproveitamento e aprendizado, já que foi possível conhecer a situação epidemiológica de mortalidade do município de Criciúma e identificar as estratégias para a redução desta variável.

Palavras-chave: mortalidade neonatal, Sistema Único de Saúde, Enfermagem.

\section{Abstract}

\section{Leading causes of neonatal death in Criciúma and the role of nurses to prevent them}

According to the Ministry of Health, the neonatal mortality has become a major component of infant mortality from the late 80's. Considering this fact, the objective of this study was to identify the main causes of neonatal mortality in the town of Criciúma/SC between 2006 and 2010, and understand the role of nurses in preventing this mortality. Data were collected by analyzing death certificates; Mortality Reporting System (MRS) data, and medical records of pregnant women in health clinics where they received prenatal care. It was found that there were 119 neonatal deaths with residents of Criciúma, being $72.2 \%$ occurred in the early neonatal period and $27.7 \%$ in the late neonatal period. We identified 47 different causes, and

Artigo recebido em 19 de dezembro de 2011; aceito em 29 de outubro de 2012 Endereço para correspondência: Aline Gabriel Lima, Rua João Marcelo, 380, 88820-000 Içara SC, Tel: (48) 9936-7338, E-mail: lynynha_2@hotmail.com 
$64 \%$ were considered preventable by interventions in the Unified Health System (UHS). Analyzing the records of pregnant women, $44.6 \%$ were not found, only $29.8 \%$ had notes from other professionals, and $25.6 \%$ had no changes. The proposed study was of great use and learning, as it was possible to know the epidemiological mortality situation of the town of Criciúma and identify strategies for reducing this variable.

Key-words: neonatal mortality, Unified Health System, Nursing.

\section{Resumen}

\section{Las principales causas de muerte neonatal en Criciúma y el papel del enfermero para prevenirlas}

Según el Ministerio de Salud, la mortalidad neonatal se ha convertido en el principal componente de la mortalidad infantil a partir de la década de los 80 . Teniendo en cuenta este hecho, el objetivo de este estudio fue identificar las principales causas de mortalidad neonatal en la ciudad de Criciúma - SC entre 2006 y 2010, y comprender el papel del enfermero en relación a la prevención de esas muertes. Los datos fueron recogidos a partir del análisis de los certificados de defunción, datos del Sistema de Informaciones sobre Mortalidad (SIM), y los registros médicos de las mujeres embarazadas en los centros de salud donde recibieron atención prenatal. Se observó que se registraron 119 muertes de recién nacidos entre los residentes de Criciúma, siendo que el 72,2\% se produjeron en el período neonatal precoz y el $27,7 \%$ en el período neonatal tardío. Se identificaron 47 diferentes causas, y el 64\% se consideraron prevenibles mediante intervenciones en el Sistema Único de Salud (SUS). Analizando el registro de las mujeres embarazadas, el 44,6\% no se encontraron, sólo el 29,8\% había apuntes de otros profesionales, y el 25,6\% no tuvo cambios. El estudio propuesto fue de gran utilidad y aprendizaje, ya que se pudo conocer la situación epidemiológica de mortalidad de la ciudad Criciúma e identificar estrategias para la reducción de esta variable.

Palabras-clave: mortalidad neonatal, Sistema Único de Salud, Enfermería.

\section{Introdução}

Entre os 130 milhóes de crianças que nascem no mundo anualmente, cerca de 4 milhóes morrem no período neonatal, proporçáo esta que pode variar de acordo com a taxa de mortalidade global. Podem ocorrer variaçóes no risco diário destas mortes e esse risco é considerado maior na primeira semana de vida [1].

A taxa de mortalidade neonatal é um indicador de saúde que, quando se encontra elevado pode indicar más condiçốes de vida e dificuldade de acesso aos serviços de saúde, tanto no período pré-natal como no atendimento hospitalar [2]. A maioria das causas de óbitos neonatais é determinada por causas perinatais, como baixo peso ao nascer, prematuridade, problemas relacionados ao parto e ao pós-parto imediato, precariedade nos serviços de saúde de pré-natal e de parto. Nesse ponto, acredita-se que muitas delas são consideradas preveníveis ou redutíveis a partir de um acompanhamento adequado da gestaçáo e parto, e outras ainda podem ser consideradas evitáveis por meio de diagnóstico e intervenção precoce $[3,4]$.

A mortalidade neonatal resulta de diversos determinantes biológicos, socioeconômicos e rela- cionados à atenção à saúde, sendo que cada agrupamento destes fatores apresentam interferência sobre o outro, permitindo uma melhor compreensão de sua importância [5]. Porém, apesar da grande ocorrência de óbitos neonatais, se houver um sistema de saúde organizado e hierarquizado, com os locais de assistência em boas condiçóes de estrutura, atendimento, e humanizaçáo no cuidado, as chances de sobrevida de um neonato aumentariam circunstancialmente [6].

A maioria das causas deste tipo de óbito pode ser prevenida através de condutas adequadas no controle da gravidez, e também de atenção ao parto. Desse modo é de suma importância que a equipe de enfermagem esteja sempre atualizada com relação aos dados e informaçóes acerca da mortalidade neonatal, para que assim colabore com a redução deste indicador de saúde juntamente com a sociedade e as instituiçóes de saúde públicas e privadas. Para que isso aconteça é necessário que o enfermeiro esteja preparado e tenha também conhecimento profissional para encontrar a forma mais adequada de prevenir a ocorrência de óbitos neonatais em sua comunidade ou instituição de saúde [7].

Diante destes e outros questionamentos referentes às causas de mortalidade neonatal e o papel 
do enfermeiro frente a esta problemática, este estudo teve como objetivo identificar as principais causas de mortalidade neonatal no município de Criciúma/ SC, no período de 2006 a 2010, e compreender o papel do enfermeiro frente a prevenção das mesmas, para que esse assunto de grande importância para a saúde pública pudesse ser melhor compreendido e a partir disso desenvolver estratégias inovadoras para a redução do número de óbitos neonatais do município estudado.

\section{Material e métodos}

O estudo teve como abordagem metodológica a quantitativa, sendo o tipo da pesquisa documental exploratória, pelo fato de que foram analisadas as declaraçóes de óbitos dos neonatos residentes em Criciúma/SC no período de 2006 a 2010, e estas causas, por sua vez, foram exploradas para melhor compreendê-las. A pesquisa teve início somente após o parecer de aprovação do Comitê de Ética em Pesquisa da Universidade do Extremo Sul Catarinense, com o projeto no 191/2011, e foi colocada em prática respeitando os princípios da Resolução 196/96 do Conselho Nacional de Pesquisa - CONEPE. O local do estudo foi o município de Criciúma, localizado no sul do estado de Santa Catarina, utilizando-se os dados de mortalidade neonatal oriundos da Vigilância Epidemiológica do atual município e da Secretaria de Saúde do Estado de Santa Catarina, além das Unidades de Saúde que foram visitadas a fim de explorar as evoluçóes realizadas pelo enfermeiro nos prontuários das gestantes realizadas durante o seu pré-natal.

O estudo foi realizado sobre a população total de óbitos neonatais dos residentes do município de Criciúma no ano de 2006 a 2010, mediante as declaraçóes de óbito, que totalizaram 119 óbitos. Os dados para a pesquisa foram coletados por meio de consulta ao banco de dados da vigilância epidemiológica, tratando-se neste caso, das declaraçóes de óbito (DO); do Sistema de Informação de Mortalidade (SIM) do SUS; e por meio da análise dos prontuários das gestantes na Unidade de Saúde onde realizaram o pré-natal. Foram analisados somente os prontuários das máes dos RNs que foram a óbito consequente das três causas mais frequentes.

Os dados obtidos na coleta foram devidamente tabulados, analisados e avaliados pelo programa de estatística SPSS versão 18.0, utilizando-se da análise de frequências. Após realizar a análise estatística, a base de dados foi transferida para o programa $\mathrm{Mi}$ crosoft Excel para construção de gráficos e tabelas e, assim, confrontados com a literatura científica. A análise foi realizada com interpretação dos dados fundamentada na revisão de literatura encontrada e por meio da realizaçáo de um estudo estatístico dos aspectos epidemiológicos encontrados nas DO e no SIM, e no caso dos prontuários, realizou-se uma análise simples dos dados obtidos, através da ordenação, classificação e análise final.

\section{Resultados}

De acordo com os dados do Sistema de Informação sobre Mortalidade do Ministério da Saúde, ocorreram 119 óbitos neonatais nos últimos cinco anos com residentes de Criciúma. Na Figura I observa-se um pequeno aumento entre os anos 2006 e 2007, em seguida uma redução até o ano de 2009, sendo que em 2010 houve novamente uma frequência de óbitos um pouco maior. Em 2011 já ocorreram 17 óbitos até fim do mês de outubro, representando que o índice ainda permanece abaixo do último ano, e espera-se que permaneça nessa média até o fim.

Analisando o coeficiente de mortalidade neonatal de cada ano observou-se que o maior foi de 11,3/1000 NV em 2007, e o menor foi de 6,89/1000 NV em 2009, representando uma grande queda no período de 2 (dois) anos o que é considerado um fator positivo. Já no Estado de Santa Catarina a média de nascidos vivos ficou entre 82.000 e 85.500 . O ano que ocorreu mais óbitos neonatais foi o de 2006, com 741 óbitos, que foram reduzindo gradativamente ao longo dos anos, chegando a 611 óbitos em 2010. O maior índice foi no ano de 2007, com 8,68/1000 NV, e o menor em 2010, com 7,22/1000, apresentando uma regressáo gradativa a partir do ano de 2008 .

Figura I - Frequência dos óbitos neonatais dos residentes de Criciúma por ano de ocorrência (2006 a 2010).

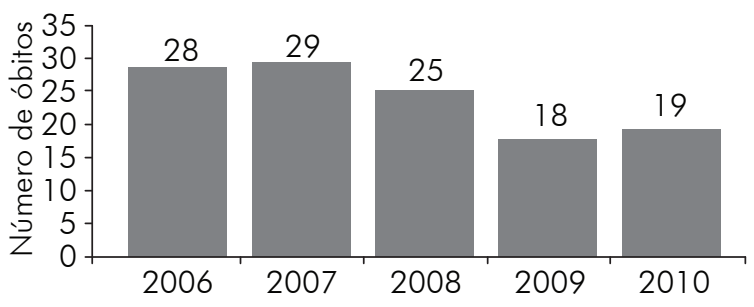

Fonte: Ministério da Saúde - Sistema de Informação sobre Mortalidade (SIM) - 2011 
Dos 119 óbitos ocorridos nos últimos cinco anos com os residentes de Criciúma, revelaram-se 47 causas diferenciadas. As que mais se destacaram foram: imaturidade extrema $(15,13 \%)$; septicemia bacteriana não especificada do recém-nascido $(15,13 \%)$; e síndrome da angústia respiratória do recém-nascido $(14,28 \%)$.

Das 47 causas encontradas 64\% são consideradas evitáveis por intervenções no âmbito do Sistema Único de Saúde do Brasil (SUS), como observado na Tabela I. Nesta as causas foram dispostas de acordo com a Classificação Estatística Internacional de Doenças e Problemas Relacionados à Saúde - 10ª Revisão, pois a descrição das mesmas deixaria a tabela muito extensa.

Tabela I - Classificação dos óbitos neonatais dos residentes de Criciúma por fator de prevenção de acordo com o manual de vigilância e do comitê de prevenção do óbito infantil e fetal - Ministério da Saúde - 2009.

\begin{tabular}{|c|c|c|}
\hline Classificação & $N^{\circ}$ (CID 10) & $\%$ \\
\hline $\begin{array}{l}\text { Redutíveis por ação de } \\
\text { imunoprevenção }\end{array}$ & 1 (G00.0) & 2,1 \\
\hline $\begin{array}{l}\text { Reduzíveis por } \\
\text { adequada atenção à } \\
\text { mulher na gestação } \\
\text { e parto e ao recém- } \\
\text {-nascido }\end{array}$ & 0 & 0 \\
\hline $\begin{array}{l}\text { Reduzíveis por } \\
\text { adequada atenção à } \\
\text { mulher na gestação }\end{array}$ & $\begin{array}{l}12 \text { (P07.2; P01.1; } \\
\text { P07.0; P55.0; P07.3; } \\
\text { P00.0; P05.0; P05.9; } \\
\text { P02.7; P02.9; P00.8; } \\
\text { P03.9) }\end{array}$ & 25,6 \\
\hline $\begin{array}{l}\text { Reduzíveis por } \\
\text { adequada atenção à } \\
\text { mulher no parto }\end{array}$ & 3 (P20.9; P21.9; P24.0) & 6,5 \\
\hline $\begin{array}{l}\text { Reduzíveis por ade- } \\
\text { quada atenção ao } \\
\text { recém-nascido }\end{array}$ & $\begin{array}{l}14 \text { (P25.1; P28.5; } \\
\text { P51.0; P26.9; P36.5; } \\
\text { P28.9; P38; P54.9; } \\
\text { P37.8; Q00.0; P23.9; } \\
\text { P77; P22.0; P36.9) }\end{array}$ & 29,8 \\
\hline
\end{tabular}

\begin{tabular}{lll}
\hline $\begin{array}{l}\text { Reduzíveis por ações } \\
\text { adequadas de diag- } \\
\text { nóstico e tratamento }\end{array}$ & 0 \\
\hline $\begin{array}{l}\text { Reduzíveis por ações } \\
\text { adequadas de promo- }\end{array}$ & 0 \\
$\begin{array}{l}\text { ção à saúde, vincula- } \\
\text { das a ações adequa- }\end{array}$ & & \\
$\begin{array}{l}\text { das de atenção à } \\
\text { saúde }\end{array}$ & 1 (P96.0) & 2,1 \\
\hline $\begin{array}{l}\text { Causas de morte mal } \\
\text { definidas }\end{array}$ & Q79.0; Q01.2; & \\
\hline & Q91.03; Q33.6; R95; & 33,9 \\
& Q05.4; L01.0; Q79.9; & \\
$\begin{array}{lll}\text { Demais causas (não } \\
\text { claramente evitáveis) }\end{array}$ & Q61.3; Q87.8; Q91.7; & \\
& Q07.9; Q32.1; Q89.9) & \\
\hline TOTAL & 47 & 100 \\
\hline Font: Dados da Pesquisa & 2011 & \\
\end{tabular}

Fonte: Dados da Pesquisa - 2011

Os locais de ocorrência variaram entre hospitais $(94,92 \%)$, maternidades $(1,68 \%)$, domicílios (1,68\%), clínicas $(0,84 \%)$, e vias públicas $(0,84 \%)$. A maioria deles $(98,28 \%)$ ocorreu no estado de Santa Catarina, sendo que 1,68\% ocorreram fora do estado, lembrando que a residência dos neonatos é Criciúma.

As mortes em via pública $(0,84 \%)$ ou domiciliares $(1,68 \%)$ ocorreram com menor frequência, sendo justificadas por dificuldade de acesso aos serviços de saúde, ou a ocorrência de um parto de emergência com complicaçóes sem assistência qualificada no momento.

O horário de ocorrência dos óbitos foi bastante variado, porém para melhor compreendê-los, os mesmos foram classificados por horários de plantões, sendo das 07:00h às $13: 00 \mathrm{~h}$, das $13: 00 \mathrm{~h}$ às $19: 00 \mathrm{~h}$, e das 19:00h às 07:00h. O intervalo de plantáo de maior ocorrência de óbito em quatro anos foi o de 12 horas, representando um maior índice em 2008,

Tabela II - Mortalidade neonatal segundo local de ocorrência dos óbitos dos residentes de Criciúma nos anos de 2006 a 2010.

\begin{tabular}{|c|c|c|c|c|c|c|c|c|c|c|c|c|}
\hline \multirow[t]{2}{*}{ Local de Ocorrência } & \multicolumn{2}{|c|}{2006} & \multicolumn{2}{|c|}{2007} & \multicolumn{2}{|c|}{2008} & \multicolumn{2}{|c|}{2009} & \multicolumn{2}{|c|}{2010} & \multicolumn{2}{|c|}{ Total } \\
\hline & $\mathrm{N}^{\circ}$ & $\%$ & $\mathrm{~N}^{\circ}$ & $\%$ & $\mathrm{~N}^{\circ}$ & $\%$ & $\mathrm{~N}^{\circ}$ & $\%$ & $\mathrm{~N}^{\circ}$ & $\%$ & $\mathrm{~N}^{\circ}$ & $\%$ \\
\hline Hospital & 27 & 22,68 & 28 & 23,52 & 25 & 21 & 16 & 13,44 & 17 & 14,28 & 113 & 94,92 \\
\hline Maternidade & - & - & - & - & - & - & 1 & 0,84 & 1 & 0,84 & 2 & 1,68 \\
\hline Clínica & - & - & - & - & - & - & - & - & 1 & 0,84 & 1 & 0,84 \\
\hline Via Pública & 1 & 0,84 & - & - & - & - & - & - & - & - & 1 & 0,84 \\
\hline Domicílio & - & - & 1 & 0,84 & - & - & 1 & 0,84 & - & - & 2 & 1,68 \\
\hline Total & 28 & 23,52 & 29 & 24,36 & 25 & 21 & 18 & 15,12 & 19 & 15,96 & 119 & 100 \\
\hline
\end{tabular}

Fonte: Declaração de Óbito 
com $64 \%$ dos óbitos ocorridos neste horário. Foi possível observar também que em ordem de ocorrência, o plantão das 07:00h às 13:00h é o que foi registrado menor ocorrência de óbitos nos últimos 5 anos, antecedendo o das 13:00h às 19:00h, que foi maior apenas no ano de 2007, com 44,8\% dos óbitos neste horário.

Referente ao período de ocorrência dos óbitos, o neonatal precoce prevaleceu durante os cinco anos pesquisados, porém diminuiu com o passar dos anos, de 89,3 \% em 2006 para 52,6\% em 2010. Já os óbitos ocorridos no período neonatal tardio apresentou um crescimento considerável no decorrer dos anos, passando de 10,7\% do total de óbitos no ano de 2006 para 47,4\% no ano de 2010, um aumento de $36,7 \%$ em cinco anos.

Em relação à raça/cor dos neonatos, a que mais prevalece nos óbitos é a cor branca, sendo a mais prevalente em todos os anos. Já referente ao sexo, o de maior ocorrência de óbitos foi o masculino, prevalecendo nos anos de 2006 a 2009, exceto em 2010 , no qual prevalece o sexo feminino com $63,2 \%$ dos óbitos ocorridos neste ano.

Referente ao peso ao nascer dos neonatos, a média variou entre $1.421 \mathrm{~g}(\mathrm{DP} \pm 998,86)$, em 2008, e 1.748,3g (DP $\pm 1087,70)$ em 2006. Os pesos mínimos variaram de $375 \mathrm{~g}$ em 2008 e 1.006,7 em 2010, sendo que o peso máximo encontrado nos cinco anos foi de 3.950g em 2007 (Figura 2). Analisando a média dos pesos, os neonatos são classificados como recém-nascido de extremo baixo peso $(<1.000 \mathrm{~g})$, muito baixo peso $(<1.500 \mathrm{~g})$ e recém-nascido de baixo peso $(<2.500 \mathrm{~g})$, características estas que contribuem para o óbito neonatal, pois o peso ao nascer é considerado um fator de risco para este tipo de óbito.

Figura 2 - Mortalidade neonatal segundo peso ao nascer dos residentes de Criciúma nos anos de 2006 a 2010.

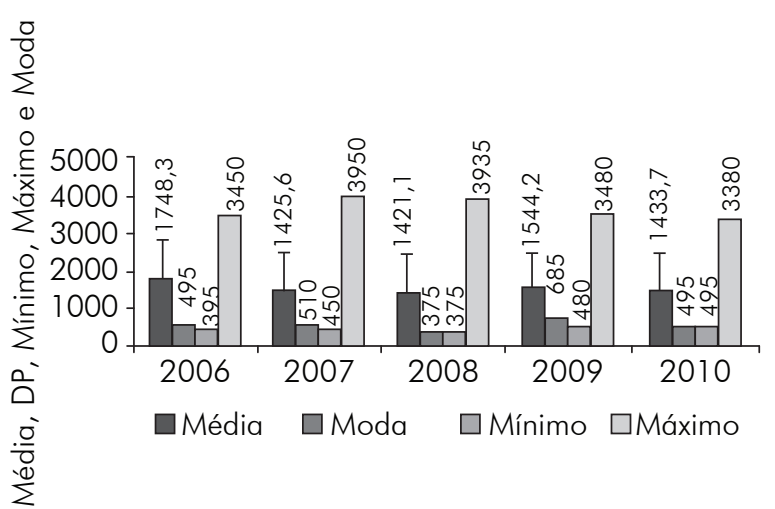

Referente à idade gestacional foi observado que a maioria dos neonatos é classificada como prematuridade extrema (22 a 31 semanas de gestaçáo), fator este considerado como risco para a mortalidade neonatal de acordo com diversas literaturas, principalmente quando associado ao baixo peso ao nascer.

Quanto ao perfil das mães dos neonatos, verificou-se que a média de idade variou entre 23,6 anos em 2006 (DP \pm 5,88 anos), e 35,46 anos em 2007 (DP $\pm 7,32$ anos). A menor idade registrada foi de 15 anos em 2006 e 2007, e a maior foi 43 em 2007. Em referência à escolaridade materna, prevaleceu de 0 a 11 anos de estudo, representando alto índice em todos os anos e um aumento de 34\% nos últimos cinco, representando que as mães possuem pelo menos o ensino fundamental completo ou incompleto como escolaridade.

Com relação ao tipo de parto dos neonatos, o cirúrgico, ou cesariana, teve um crescimento de $43,2 \%$ nos últimos cinco anos, prevalecendo sobre o parto vaginal a partir do ano de 2009 , sendo que este último iniciou com 57,1\% em 2006 e reduziu para $21,1 \%$ em 2010 .

Além de compreender a epidemiologia de mortalidade neonatal do município, o papel do enfermeiro frente a esta problemática também foi investigado. Procuramos analisar as evoluçóes desse profissional em relação ao pré-natal das gestantes nas unidades de saúde. Foram procurados 47 prontuários, e destes $44,6 \%$ não foram encontrados nas unidades, justificados pelo fato de talvez a gestante morar só por um tempo no local ou ter mudado de casa, bairro ou cidade. Já os prontuários encontrados, que totalizaram $55,4 \%$ dos procurados, não possuíam evoluçáo, sendo que $25,6 \%$ não possuíam anotaçóes, porque o pré-natal foi realizado em outra unidade, e as anotaçóes dos 29,8\% eram de outros profissionais e não do enfermeiro.

\section{Discussão}

De 1990 a 2007 a taxa de mortalidade infantil (TMI) no Brasil apresentou tendência de queda, passando de 47,1/1000 NV em 1990 para 19,3/1000 NV em 2007. Já a taxa de mortalidade neonatal, que indica o risco de um nascido vivo morrer de 0 a 27 dias de vida, vem mantendo níveis cada vez mais elevados. Totalizou em 2007 13,2/1000 $\mathrm{NV}$, um grande índice quando comparada à taxa de outros países como Argentina (10/1000), Chile (5/1000), Canadá (3/1000) e França (2/1000). 
Esta taxa, por sua vez, reflete de maneira geral as condiçốes socioeconômicas e de saúde da mãe, bem como inadequada assistência pré-natal ao parto e ao recém-nascido [8].

Nas últimas décadas, a mortalidade neonatal precoce vem aumentando relativamente sua participação na mortalidade infantil, sendo que a desigualdade social e econômica pode ser o principal determinante no aumento do risco de morte nesse período, bem como a conjunção de fatores biológicos, socioeconômico e assistencial, sendo estes últimos relacionados a atenção à gestante e ao recém-nascido $[9,10]$.

Em virtude disto, percebe-se a importância de uma assistência humanizada e responsável por parte dos profissionais de saúde para com as gestantes no pré-natal e com os neonatos após o nascimento, para que estes índices encontrados a partir dos dados da pesquisa reduzam cada vez mais, contribuindo para a redução da mortalidade a nível nacional e municipal.

A maioria das causas de óbitos neonatais é determinada por causas perinatais, como baixo peso ao nascer, prematuridade, problemas relacionados ao parto e ao pós-parto imediato, precariedade nos serviços de saúde de pré-natal e de parto [3]. Referente às três causas mais frequentes encontradas durante a realizaçáo do estudo, a imaturidade, que representou 15,13\% dos óbitos, pode levar à disfunção em qualquer órgão ou sistema corporal, e o neonato prematuro pode, além disso, sofrer comprometimento ou intercorrências maiores ao longo do seu desenvolvimento [11].

Por outro lado, a septicemia, que também representou $15,13 \%$ das causas dos óbitos é definida como uma síndrome clínica caracterizada por uma resposta inflamatória sistêmica na presença de uma infecção suspeita ou confirmada [12]. A síndrome da angústia respiratória do recém-nascido, que representou $14,28 \%$ das causas, é considerada uma das mais frequentes de sintomatologia respiratória e de óbito em recém-nascidos prematuros, sendo este distúrbio decorrente da produção insuficiente de surfactante pulmonar e má adaptação da vida extrauterina [13].

Das 47 causas encontradas 64\% são consideradas evitáveis por intervenções no âmbito do Sistema Único de Saúde do Brasil (SUS), como já comentado anteriormente. Nesse contexto, autores discutem que, em ambos os componentes da mortalidade infantil (neonatal e pós-neonatal), uma importante parcela é de responsabilidade atribuída aos serviços de saúde. Acredita-se que algumas medidas sanitárias adequadas e serviços de saúde acessíveis e de boa qualidade a toda a populaçáo, podem atuar na redução da mortalidade infantil em ambos os componentes [3].

Nesse ponto é possível concordar com o autor, sendo que se houver empenho por parte dos prestadores de serviços de saúde no que se refere à melhoria da assistência e acessibilidade, a adesão a estes serviços seria maior e mais eficaz na reduçáo da mortalidade. Deduz-se também que, além de realizar ações de prevenção durante a gestação, é necessário, por parte dos profissionais de saúde, que após o parto a prevenção ainda continue sendo realizada com humanizaçáo, paciência, e cuidado com o neonato, contribuindo cada vez mais para a redução dos óbitos nesse período.

Justificando as mortalidades ocorridas com os residentes de Criciúma no ambiente hospitalar que representou $94,92 \%$ dos óbitos, autores refletem que a reduzida oferta de serviços, como a ausência de atendimento pediátrico na sala de parto, pode influenciar no aumento da mortalidade neonatal precoce hospitalar, enfocando a qualidade dos serviços prestados [9].

Outros já refletem que a maior ocorrência de óbitos hospitalares acontece no período neonatal precoce, do nascimento até o $6^{\circ}$ dia de vida, pelo fato de este ser o período em que o neonato permanece mais tempo neste ambiente para se submeter aos primeiros cuidados pós-parto. Sendo assim, é possível perceber a estreita relação entre os óbitos infantis e a assistência ao parto e nascimento, que predomina no ambiente hospitalar em nosso país, com exceçóes em algumas localidades que ainda não possuem este tipo de assistência [8].

Não foi encontrado nenhum estudo atual que indicasse influência do horário de nascimento para o óbito neonatal, somente um que discutia que na $\mathrm{Su}$ écia foi encontrado um alto risco de morte neonatal em nascimentos no período noturno, e no México e Alemanha, em nascimentos prematuros, o horário da noite também representou alto risco de morte em relação ao horário diurno [7]. Porém tivemos por conclusáo que, embora o período noturno tenha um quadro reduzido de profissionais e a jornada de trabalho mais longa, os dados indicam que esse não é um fator significativo para número de óbitos ocorridos nesse período.

Confrontando com os dados do estudo, foi no horário noturno que ocorreu mais óbitos em quatro 
anos dos cinco estudados, representando que esta variável representou risco para os óbitos ocorridos. Mas o que justificaria isto? Número reduzido de profissionais? De assistência humanizada? De cuidado? Jornada de trabalho extensa? Exaustão dos profissionais? Déficit na assistência?. Isto é importante para que se reflita e se busque mais pesquisas para o entendimento de tal problemática e, assim, propor estratégias de melhoria da assistência ao neonato e para que muitas das mortes nesse horário sejam evitadas.

Analisando a média dos pesos dos neonatos, os mesmos são classificados como recém-nascido de extremo baixo peso $(<1.000 \mathrm{~g})$, muito baixo peso $(<1.500 \mathrm{~g})$ e recém-nascido de baixo peso $(<2.500$ g), características estas que contribuem para o óbito neonatal, pois o peso ao nascer é considerado um fator de risco para este tipo de óbito. De acordo com a literatura os nascimentos de muito baixo peso representam uma grande parte dos óbitos neonatais, o que destaca que é importante se conhecer melhor as características dos subgrupos de peso e idade gestacional, bem como seus fatores de risco, para que estes óbitos sejam mais bem compreendidos, facilitando as estratégias para sua redução [14].

O peso abaixo do normal geralmente se associa à prematuridade como um risco de óbito neonatal, sendo que esta hipótese pode ser confirmada quando observamos que a prematuridade extrema foi a idade gestacional que prevaleceu durante os cinco anos pesquisados.

A prematuridade e o baixo peso ao nascer são apresentados na literatura como importantes fatores de risco para o óbito neonatal. Porém, estas duas variáveis não podem ser analisadas isoladamente, mas como mediadoras de outros determinantes, como o nível socioeconômico materno, o acesso aos serviços de saúde e a qualidade dos mesmos, dentre outros [2]. Em virtude disto, constata-se que estas duas variáveis juntas foram fatores de risco comprovados para os óbitos ocorridos com os residentes de Criciúma nos últimos cinco anos, como diz a literatura.

Quanto às características maternas como idade e escolaridade foi encontrado que as idades que mais se destacaram durante os anos foi entre $18 \mathrm{e}$ 25 anos, idades estas consideradas favoráveis para se engravidar segundo algumas literaturas. Já idades mais elevadas, como aquelas acima de 35 anos, são consideradas de risco, como já citado, e justificam-se pelo fato de que, ultimamente o casal procura primeiro organizar a vida financeira e profissional para depois pensar em constituir família.

Referente à idade da mãe, a literatura traz que existem dois períodos da vida da mulher em que é observado maior ocorrência de óbitos perinatais: quando as gestaçóes ocorrem antes dos 15 e também após os 40 anos de idade. Nesses períodos as causas encontradas com mais frequência são a prematuridade, o baixo peso ao nascer, o abortamento espontâneo e as malformaçóes fetais [15]. Em virtude disso, percebemos que a idade da máe neste estudo náo foi considerada um risco para os óbitos neonatais dos residentes de Criciúma.

Quanto ao nível de escolaridade materno, de acordo com o estudo realizado, foi considerado baixo, o que pressupóe um fator de risco para os óbitos neonatais [5]. O nível de escolaridade geralmente reflete as condições socioeconômicas do indivíduo, pois quanto mais provida de conhecimento for a mulher, mais cuidados ela tem consigo mesma em relaçáo à sua saúde, seus hábitos alimentares e também mais responsabilidade quanto ao planejamento familiar, o que contribui para o desenvolvimento de uma boa gestação e, consequentemente, uma boa evolução após o parto, reduzindo o risco de óbitos neonatais.

Quanto ao tipo de parto, observou-se que o cesáreo teve uma grande vantagem sobre o parto vaginal nos cinco anos pesquisados. A literatura revela que a cesariana representa um fator de proteção para os RN's prematuros e de baixo peso, diminuindo assim o risco de morte nesses casos, já o parto vaginal representaria maior risco [1].

Nesse sentido, analisando que, no presente estudo, a maioria dos neonatos foi classificada como extremo baixo peso e prematuridade extrema, o que nos levou a uma justificativa para esse aumento súbito do parto cirúrgico. Porém este grande aumento também pode ser justificado pelo medo, conveniência e desinformação por parte da mãe, que muitas vezes receia as consequências de um parto por via vaginal, considerando este como uma experiência arriscada, sugerindo que o ato cirúrgico é um modo para se evitar a dor [16].

Da mesma forma, existem muitos profissionais de saúde que defendem o parto vaginal como primeira escolha, considerando as vantagens que este tipo de parto tem sobre a cesariana, tendo em vista que o corpo da mulher foi preparado para isto. Sendo assim, observa-se a necessidade de se realizar açôes educativas voltadas às mulheres e à população em geral enfatizando as vantagens e desvantagens 
do parto vaginal e do procedimento cirúrgico, a cesariana; as práticas assistências disponíveis; e os métodos de alívio da dor, para que assim seja reforçada a opção pelo parto normal [16,17].

Para avaliar o papel do enfermeiro frente à prevenção mortalidade neonatal no município estudado, foram visitadas 27 unidades de saúde de tal município a fim de identificar evoluções realizadas pelo enfermeiro no prontuário das gestantes cujos neonatos foram a óbito proveniente de uma das três causas mais frequentes identificadas nos últimos cinco anos. Foram identificados 53 prontuários a serem procurados, porém seis não continham o endereço nos dados das DO's, não sendo possível sua procura. Portanto foram procurados 47 prontuários buscando identificar as evoluçóes do enfermeiro voltadas ao pré-natal das gestantes.

Como já citado os resultados encontrados não foram positivos, pois os 55,6\% dos prontuários encontrados náo continham nenhuma evoluçáo por parte do enfermeiro voltado ao pré-natal da gestante. Nesse ponto percebe-se que a maior ferramenta de trabalho da enfermagem não é colocada em prática pelos profissionais da atenção primária de Criciúma.

Referente às evoluçôes, autores discutem que os registros no prontuário do paciente, feitos pela equipe de enfermagem, devem refletir as condições biopsico-sócio-espirituais do paciente, sendo o local onde são relatadas todas as ocorrências relacionadas a ele, de forma que seja possível a elaboração de planos assistenciais, a implementação de ações que visem melhorias nos resultados operacionais, e a continuidade de cuidados referentes ao seu estado de saúde [18].

Em virtude disso podemos identificar que a qualidade da assistência prestada pelos enfermeiros de Criciúma nos últimos cinco anos é caracterizada de forma negativa, pelo fato de que não foi encontrada nenhuma evoluçáo dos mesmos referente ao pré-natal das gestantes. Assim percebe-se que, além de elaborar uma evoluçáo de qualidade, o enfermeiro precisa também qualificar a assistência pré-natal fornecida às gestantes em nosso município, pois é neste momento que muitas causas de futuros óbitos neonatais ou maternos podem ser identificadas a tempo de propor medidas adequadas de prevenção.

\section{Conclusão}

O presente estudo buscou identificar os índices de mortalidade neonatal dos residentes de Criciúma nos anos de 2006 a 2010, bem como as principais causas que influenciaram nestes óbitos e os locais de ocorrência dos mesmos. A partir da obtenção das causas, as mesmas foram analisadas pelo grau de evitabilidade, e a partir daí outro objetivo foi procurar compreender como o profissional enfermeiro enfrenta tal problemática através da assistência prestada à saúde da gestante e do neonato, bem como identificar os registros desta assistência nos prontuários das gestantes cujos neonatos foram a óbito proveniente de uma das três causas mais frequentes, ocorridas nos anos pesquisados no estudo.

Foi de grande aproveitamento e aprendizado a realização desta pesquisa, pois foi possível conhecer a situação epidemiológica de mortalidade do município e identificar as estratégias para a redução desta variável a partir dos estabelecimentos de saúde envolvidos, e do Comitê Municipal de Prevenção do Óbito Materno Infantil. Não foi possível um aprofundamento maior no assunto pesquisado por falta de tempo para a continuidade da pesquisa, porém muitas lacunas foram encontradas para futuros estudos e investigaçóes referentes ao assunto pesquisado, tanto a nível municipal como estadual.

A mortalidade neonatal é uma das mais intrigantes causas do processo da interrupção da vida. A riqueza de um pré-natal e as características do ciclo gravídico, tais como o seu início precoce, o acompanhamento do seu curso até o nascimento, e as alteraçóes fisiológicas e psicológicas despertam curiosidade e geram uma preocupação considerável na assistência sobre os processos evolutivos dessa etapa, pois muitos avanços serão necessários para compreender melhor as causas que levam ao óbito neonatal. No entanto, muito ainda está por vir. Diversos grupos de profissionais de saúde deverão utilizar as mais variadas metodologias para que tenhamos uma melhor compreensão dos processos envolvidos que levam ao óbito. Espera-se que o conhecimento aprofundado das principais causas envolvidas permita o desenvolvimento de assistência mais eficaz e o controle para a prevenção de óbitos neonatais em nosso município. Sugere-se que mais pesquisas sejam realizadas para contribuir com a redução da taxa do óbito neonatal.

\section{Referências}

1. Zanini RR, Moraes AB, Giugliane ERJ, Riboldi J. Determinantes contextuais da mortalidade neonatal no Rio Grande do Sul por dois modelos de análise. Rev Saúde Pública 2011;45(1): 79-89. 
2. Paulucci RS, Nascimento LFC. Mortalidade neonatal em Taubaté: um estudo caso-controle. Rev Paul Pediatr 2007;25(4):358-63.

3. Caldeira AP, França E, Perpétuo IHO, Goulart EMA. Evolução da mortalidade infantil por causas evitáveis, Belo Horizonte, 1984-1998. Rev Saúde Pública 2005;39(1):67-74.

4. Lima EFA, Souza, AI, Primo CC. Mortalidade Neonatal em Serra, Espírito Santo, 2001-2005. Rev Enferm UERJ 2008;16(2):162-7.

5. Ribeiro AM, Guimarães MJ, Lima MC, Sarinho SW, Coutinho SB. Fatores de risco para mortalidade neonatal em crianças com baixo peso ao nascer. Rev Saúde Pública 2009;43(2):246-55.

6. Pinheiro CEA, Peres MA, D’ Orsi E. Aumento na sobrevida de crianças de grupos de peso baixo ao nascer em Santa Catarina. Rev Saúde Pública 2010;44(5):77684.

7. Helena ETS, Souza CA, Silva CA. Fatores de risco para mortalidade neonatal em Blumenau, Santa Catarina: linkage entre bancos de dados. Rev Bras Saúde Mater Infant 2005;5(2):209-17.

8. Brasil. Ministério da Saúde. Manual de vigilância do óbito infantil e fetal e do comitê de prevenção do óbito infantil e fetal. Brasília: Ministério da Saúde; 2009.

9. Campos D, Loschi RH, França E. Mortalidade neonatal precoce hospitalar em Minas Gerais: associação com variáveis assistenciais e a questão da subnotificaçáo. Rev Bras Epidemiol 2007;10(2):223-38.

10. Soares ES, Menezes GMS. Fatores associados à mortalidade neonatal precoce: análise de situação no nível local. Epidemiol Serv Saude 2010;19(1):51-60.
11. Ramos HAC, Cuman RKN. Fatores de risco para prematuridade: pesquisa documental. Esc Anna Nery Rev Enferm 2009;13(2):297-304.

12. Herrmann DMML, Amaral LMB, Almeida SC. Fatores de risco para o desenvolvimento de sepse neonatal tardia em uma Unidade de Terapia Intensiva. Pediatria 2008;30(4):228-36.

13. Shepherd R. Fisioterapia em Pediatria - 3a . ed. - São Paulo: Santos Livraria; 2006. p.110-44.

14. Almeida MFB, Guinsburg R, Martinez FE, Procianoy RS, Leone CR, Marba STM, et al. Fatores perinatais associados ao óbito precoce em prematuros nascidos nos centros da Rede Brasileira de Pesquisas Neonatais. Rev Soc Boliv Pediatr 2010;49(1):48-57.

15. Senesi LG, Tristão EG, Andrade RP, Krajden ML, Oliveira Júnior FC, Nascimento DJ. Morbidade e mortalidade neonatais relacionadas à idade materna igual ou superior a 35 anos, segundo a paridade. Rev Bras Ginecol Obstet 2004;26(6):477-82.

16. Oliveira CP, Alberti LR, Petroianu A. Morbidade neonatal e materna relacionada ao tipo de parto. Ciênc Saúde Coletiva 2010;15(2):427-35.

17. Dias MAB, Domingues RMSM, Pereira APE, Fonseca SC, Gama SGN, Theme Filha MM et al. Trajetória das mulheres na definição pelo parto cesáreo: estudo de caso em duas unidades do sistema de saúde suplementar do estado do Rio de Janeiro. Ciênc Saúde Coletiva 2008;13(5):1521-34.

18. Setz VG, D’Innocenzo M. Avaliação da qualidade dos registros de enfermagem no prontuário por meio da auditoria. Acta Paul Enfermagem 2009;22(3):313-7. 